\title{
Síntomas presentes en la esclerosis múltiple: serie de casos
}

\author{
Symptoms present in multiple sclerosis: case series
}

Andrea Paola Camargo Rojas (1), Angélica María Gómez López (2), Leonardo Fabio Hernández (3), Eduardo Palacios Sánchez (4)

\section{RESUMEN}

INTRODUCCIÓN: existen síntomas presentes en la esclerosis múltiple (EM) que no están relacionados directamente con el carácter desmielinizante de los brotes, que afectan y deterioran la calidad de vida de los pacientes y que no han sido adecuadamente caracterizados en la población colombiana.

OBJETIVO: describir las características clínicas, sociodemográficas y los síntomas en los pacientes con EM del servicio de Neurología del Hospital de San José de Bogotá.

MATERIALES Y MÉTODOS: estudio de serie de casos. Pacientes consecutivos, con diagnóstico de EM (según criterios McDonald 2010) que asistieron al Hospital de San José entre agosto de 2015 y marzo de 2017. Se midieron variables sociodemográficas, clínicas y síntomas evaluados con la escala de intensidad de la fatiga, escala de depresión de Hamilton, escala de discapacidad (EDSS) y cuestionario sobre la sexualidad e intimidad en la EM. Se analizaron datos en STATA 13.

RESULTADOS: se incluyeron 65 pacientes, el $72 \%$ de sexo femenino. Síntomas presentes: fatiga (75\%), cefalea (43\%), disfunción urinaria (38\%), depresión (39\%), trastornos del sueño (32\%), disfunción anorectal $(27 \%$ ) y disfunción sexual (20\%). En depresión, el $64 \%$ presenta trastorno del sueño. El $50 \%$ de los pacientes con fatiga y depresión tenía 9 años o menos de evolución de enfermedad y 11 años o menos en los que presentan disfunción urinaria.

DISCUSIÓN Y CONCLUSIONES: es probable que la fatiga y depresión estén relacionados con mayor presentación de trastornos del sueño y disfunción sexual, evidenciándose en pacientes con mayor tiempo de evolución de la enfermedad.

PALABRAS CLAVES: Depresión; Cefalea; Esclerosis Múltiple; Fatiga; Sueño. (DeCS).

\section{SUMMARY}

INTRODUCTION: There are symptoms present in multiple sclerosis (MS), they are not directly related with the demyelinating nature of relapses, they affect and deteriorate patients' quality of life and they have not been adequately characterized in the Colombian population.

OBJECTIVE: To describe the clinical, sociodemographic and symptomatic characteristics of patients with MS in the Neurology Department of the Hospital San José de Bogotá.

MATERIALS AND METHODS: A case series study. Consecutive patients with a diagnosis of MS according to Mc Donald 2010 criteria who were attended at the Hospital de San José between August 2015 and March 2017. Sociodemographic, clinical and symptom variables were evaluated with fatigue intensity scale, Hamilton depression scale, disability scale (EDSS), and questionnaire of sexuality and intimacy in MS. Data were analyzed in STATA 13.

RESULTS: 65 patients were included. $72 \%$ female. Symptoms present: fatigue (75\%), headache (43\%), urinary dysfunction $(38 \%)$, depression (39\%), sleep disorders $(32 \%)$, anorectal dysfunction $(27 \%)$ and sexual dysfunction $(20 \%)$. In patients with depression, $64 \%$ have sleep disorders associated. $50 \%$ of patients with fatigue and depression had 9 years or less of disease progression and 11 years or less of those with urinary dysfunction.

(1) Residente IV de Neurología, Fundación Universitaria Ciencias de la Salud, Bogotá, Colombia.

(2) Especialista en Neurología, instructor asistente, Fundación Universitaria de Ciencias de la Salud, Bogotá, Colombia.

(3) Especialista en Neurología, Fundación Universitaria de Ciencias de la Salud, Bogotá, Colombia.

(4) Especialista en Neurología, instructor titular, Fundación Universitaria de Ciencias de la Salud, Bogotá, Colombia.

Recibido 22/08/17. Aceptado: 14/02/18.

Correspondencia: Andrea Paola Camargo Rojas, apcamargo@fucsalud.edu.co 
DISCUSSION AND CONCLUSIONS: Fatigue and depression are likely to be associated with increased of presentation of sleep disorders and sexual dysfunction, they are evidenced in patients with a longer evolution of the disease.

KEY WORDS: Depression; Headache; Multiple Sclerosis; Fatigue; Sleep (MeSH).

\section{INTRODUCCIÓN}

La esclerosis múltiple (EM) es una enfermedad desmielinizante, de tipo neurodegenerativo, que se presenta de preferencia en adultos jóvenes, con un promedio de edad de aparición entre 20 a 40 años (1), se considera que su fisiopatología es el resultado de la interacción de factores ambientales (2), biológicos (3), inmunológicos (4) y genéticos (5). Fue descrita inicialmente por Friedrichs en Alemania, y más adelante por Charcot (6). En el 2013 se reportó que aproximadamente 2,3 millones de personas en el mundo la padecen (7).

Los datos epidemiológicos en Latinoamérica referencian a Argentina como una zona de alta prevalencia de la enfermedad, mientras que Perú, Venezuela y Colombia se consideran países de bajo riesgo para el desarrollo de la misma (8). En Bogotá se encuentra una prevalencia de $16,25 / 100.000$ habitantes (9) y de 1,48 a $4,98 / 100.000$ habitantes en otras ciudades del país (10).

Dentro del protocolo diagnóstico de la enfermedad, se tienen en cuenta los criterios de McDonald 2010 acerca de diseminación en espacio y tiempo (11); sin embargo, es relevante considerar la posibilidad del síndrome clínico aislado, como parte de un seguimiento juicioso o posible inicio de tratamiento ante la presencia de criterios clínicos e imagenológicos adicionales (12).

El grado de discapacidad de los pacientes se evalúa con la aplicación de la escala de EDSS (13), que cuantifica la discapacidad en ocho sistemas funcionales (FS) y permite a los neurólogos asignar un nivel de sistema funcional (SFS) en cada uno de ellos. Estos son evaluados mediante los criterios de EDSS (14).

Asociado a los síntomas relacionados con los brotes de desmielinización, existe una serie de manifestaciones (15) que no guardan relación con esta fisiopatología, aunque tienen una presentación frecuente, tales como la fatiga, reportada en la literatura en el $80 \%$ de los pacientes, de los cuales alrededor de la mitad lo refieren como una de las condiciones que genera más incomodidad (16).

En cuanto a la cefalea, se puede hallar hasta en el 53,3 $\%$ de los pacientes, referida como síntoma inicial de la enfermedad o en el transcurso de la misma (17). Según los criterios internacionales establecidos (IHS), la cefalea de tipo tensión se presenta en un 37,2\%, seguida por la migraña en un $27 \%$ (18).
Los trastornos neuropsiquiátricos más relevantes son la depresión $(23,7 \%$ ) y el trastorno de sueño $(67 \%)$, siendo el insomnio de conciliación o mantenimiento el más representativo (80 \%). Adicionalmente, es importante mencionar que hasta un $2,5 \%$ de los pacientes puede presentar adicción a sustancias de abuso $(19,20)$.

De un $80 \%$ al $96 \%$ de los pacientes con EM son remitidos a consulta de urología en el curso de su enfermedad, por problemas relacionados con incontinencia urinaria, fecal o disfunción sexual, que puede darse hasta en el 60 $\%$ de los casos, sin tener una relación directa con compromiso medular por brotes de la patología de base (21). La prevalencia del estreñimiento e incontinencia fecal oscilan en torno al $40 \%$ y ameritan atención por las especialidades pertinentes (22).

En este estudio de serie de casos el objetivo principal fue realizar una descripción sociodemográfica y clínica de los pacientes con diagnóstico de EM, ampliando el interrogatorio hacia síntomas no relacionados con recaídas de lesiones desmielinizantes, los cuales no han sido reportados ampliamente en la literatura disponible en Colombia. Asimismo, el Servicio de Neurología se ha propuesto como base para estudios que puedan proponer estrategias de intervención temprana para el tratamiento multidisciplinario de la EM y que puedan mejorar la calidad de vida, el desempeño social y la estabilidad de sus relaciones interpersonales, en pro de generar bienestar en cada uno de los pacientes que se atienden en nuestra institución y, posiblemente, implementarlo a nivel nacional.

\section{MATERIALES Y MÉTODOS}

\section{Diseño}

Estudio observacional, tipo serie de casos. Pacientes mayores de 18 años con diagnóstico de EM según los criterios de McDonald 2010 (11) que asistieron a la consulta externa u hospitalización, en el Hospital Universitario de San José de Bogotá entre agosto de 2015 y marzo de 2017.

Se recogieron datos sociodemográficos, antecedentes familiares y variables clínicas, tales como: años de evolución de la enfermedad, escala de discapacidad EDSS (13) y síntomas de disfunción del tracto urinario o anorrectal. Se objetivaron síntomas como fatigabilidad por medio de la aplicación de la Escala de intensidad de la fatiga (23) (la 
cual no está validada al español, pero se utilizó la versión en castellano (24)), Escala de depresión de Hamilton (25) (validada y en versión castellana (26)), cuestionario sobre la sexualidad e intimidad en la EM (la cual no está validada al español (27)). Se determinó el tipo de cefalea basándose en la III edición de la clasificación Internacional de las cefaleas versión beta, publicada en marzo de 2013 (28). Se realizó una encuesta al paciente y sus familiares en la consulta externa o durante la hospitalización y los datos se transcribieron a una base de datos de Excel.

Esta investigación fue aprobada por el Comité de Ética de Investigación de la Fundación Universitaria de Ciencias de la Salud (FUCS).

\section{Análisis estadístico}

Se realizó con descripción de variables demográficas y clínicas por medio de frecuencias absolutas y relativas para variables cualitativas; para variables cuantitativas se emplearon medidas de tendencia central y de dispersión o medianas y rangos intercuartílicos, según la distribución normal de los datos. El análisis de los datos se realizó con el paquete estadístico STATA 13.

\section{RESULTADOS}

Un total de 65 pacientes fueron incluidos para el análisis de los datos. El $72 \%$ de los pacientes con EM son de sexo femenino. El 52 \% están solteros, divorciados o viudos. El tipo EM de recaída y remisión se presenta en 81 \%, la forma progresiva en un $15 \%$ y la variante pseudotumoral en un $3 \%$. El $4 \%$ de los pacientes presentan asociado un diagnóstico de epilepsia, como comorbilidad relevante (tabla 1).

La fatiga presentada en el $75 \%$ de los pacientes es el síntoma más frecuente, siendo de 50 el máximo puntaje encontrado con la aplicación de la escala de intensidad de la fatiga. El segundo síntoma más frecuente es cefalea, con un $43 \%$; en cuanto a los criterios IHS, la cefalea tipo tensión es más frecuente con un bajo porcentaje de presentación de migraña en el $9 \%$ de los casos (tabla 2).

Del total de la población estudiada, el $42 \%(27 / 65)$ presentan disfunción del tracto urinario; de ellos, el $98 \%$ (26/27) consultó por síntomas irritativos. La depresión se encontró en el 39 \% (25/65) de los enfermos con un compromiso moderado a severo en 23 de ellos, según la puntuación de Hamilton. Un $20 \%$ (13/65) de los pacientes presenta disfunción sexual (tabla 2).

Del total de los pacientes con fatiga, el $36 \%$ presenta trastornos del sueño, el $23 \%$ disfunción sexual y el $30 \%$ disfunción anorrectal (tabla 3). De los 25 pacientes con depresión, el $64 \%$ presenta trastorno del sueño, el $44 \%$ disfunción sexual y el $40 \%$ disfunción anorrectal (tabla 3).
Tabla 1. Características sociodemográfico y clínicas

\begin{tabular}{ll} 
Características sociodemográficas y clínicas & N: $\mathbf{6 5}(\mathbf{\%})$ \\
\hline Sexo femenino & $47(72,3)$ \\
Edad mediana (RIQ)* & $39(33-47)$ \\
Estado civil soltero & $34(52,3)$ \\
Ocupación cesante & $28(43,1)$ \\
Edad de inicio de los síntomas mediana (RIQ) & $29(20-39)$ \\
Edad de diagnóstico mediana (RIQ) & $31(23-40)$ \\
Antecedente de epilepsia focal & $3(4,62)$ \\
Antecedente familiar de EM & $7(10,8)$ \\
Subtipo de enfermedad & \\
Recaída remisión & $53(81,5)$ \\
Primaria progresiva & $6(9,2)$ \\
Secundaria progresiva & $4(6,1)$ \\
EM variante pseudotumoral & $2(3,1)$ \\
EDSS actual & $2(1-5)$ \\
Tratamiento & \\
Ninguno & $2(3,11)$ \\
Moderada eficacia & $40(61,5)$ \\
Alta eficacia & $22(33,85)$ \\
Otros & $1(1,54)$ \\
\hline Nota: RIQ: &
\end{tabular}

Nota: * RIQ: rango intercuartílico; EDSS: escala de discapacidad. Fuente: autores.

El $50 \%$ de los pacientes con depresión y fatiga tienen 9 años o menos de evolución de EM y los que presentan disfunción urinaria tiene 11 años o menos de evolución de la enfermedad (tabla 3). El 50\% de los pacientes con EM tienen 2 o menos puntos en la escala de discapacidad de EDSS (tabla 3).

\section{DISCUSIÓN}

Aunque la EM tiene una baja prevalencia en nuestro país, es una enfermedad que compromete severamente la independencia $(9,10)$, impacta en el campo laboral y conlleva un índice de desempleo que se encuentra entre el 40 y el $70 \%$ (29); adicionalmente, este abandono laboral ocurre de forma temprana en el curso de la enfermedad (30). Síntomas como la fatiga (31) y el compromiso motor disminuyen las probabilidades de conseguir o permanecer laborando (32). En los resultados, el $43 \%$ de los pacientes se encuentra en el hogar, sin una actividad laboral comprobada, algo similar a los hallazgos mencionados (29).

Podemos inferir, de este estudio una probable afectación de la vida de pareja, pues la mitad de los pacientes del estudio están solteros o divorciados; se ha encontrado que la proba- 
Tabla 2 Frecuencia de síntomas en los pacientes con EM

\begin{tabular}{|c|c|}
\hline Síntomas presentes & N: $65(\%)$ \\
\hline Fatiga & $49(75,4)$ \\
\hline Depresión & $25(38,5)$ \\
\hline Leve & $1(3,8)$ \\
\hline Moderada & $16(61,5)$ \\
\hline Severa & $7(26,9)$ \\
\hline Muy severa & $2(7,6)$ \\
\hline Trastornos del sueño & $21(32,3)$ \\
\hline Somnolencia diurna & $10(15,4)$ \\
\hline Insomnio & $20(30,8)$ \\
\hline Apnea obstructiva del sueño & $3(4)$ \\
\hline Trastorno del sueño Rem & $1(1,5)$ \\
\hline Disfunción del tracto urinario & $27(41,5)$ \\
\hline Urgencia & $13(20)$ \\
\hline Incontinencia & $15(23,1)$ \\
\hline Goteo & $8(12,3)$ \\
\hline Aumento de la frecuencia & $13(20)$ \\
\hline Retención & $1(1,54)$ \\
\hline Disfunción sexual & $13(20)$ \\
\hline Primaria & $10(76,9)$ \\
\hline Secundaria & $3(23,1)$ \\
\hline Disfunción anorrectal & $18(27,7)$ \\
\hline Cefalea & $28(43,1)$ \\
\hline Migraña & $6(9,2)$ \\
\hline Cefalea tensional & $23(35,4)$ \\
\hline
\end{tabular}

bilidad de tener o permanecer en una relación disminuye en tanto que aumentan los años de evolución de la enfermedad y el grado de discapacidad, como lo menciona Pfleger (33).

El $4 \%$ de los pacientes del estudio padece epilepsia focal, resultado levemente mayor a lo reportado en la literatura, ya que revisiones sistemáticas han reportado una incidencia de crisis del 2,3 \% y prevalencia del $3 \%$ (34); además, se ha descrito que en, la forma clínica de la EM, la escala EDSS o el sexo no son factores asociados a la aparición de crisis epilépticas (35).

En la literatura se ha descrito que la prevalencia de la fatiga está entre el $53 \%$ y el $90 \%$ (20); los pacientes la han mencionado como el peor síntoma experimentado en el $55 \%$ de los casos (16) y para el $22 \%$ ha interferido con su funcionamiento diario (20). Se presenta en mayor frecuencia en pacientes de más edad, con más tiempo de la enfermedad y en los que presentan mayor discapacidad (36). En nuestro estudio se encontró que este es el síntoma con más alta frecuencia; los pacientes tienen una media de edad y de años de enfermedad mayor en el subgrupo de los fatigados con respecto a los no fatigados y, a diferencia de otros reportes, no se evidencia relación con la mediana de la escala EDSS actual.

Hasta un $50 \%$ de los pacientes ha presentado trastornos de sueño en algún momento de la vida (37), que resulta en el aumento de la somnolencia diurna, fatiga y depresión (38), llevando a una menor calidad de vida. Se ha encontrado una prevalencia del 14 al $58 \%$ para síndrome de piernas inquietas, del 2 al $3 \%$ para trastornos del sueño REM y del 7 al $58 \%$ para apnea obstructiva del sueño (34).

Tabla 3. Síntomas presentes en la esclerosis múltiple

\begin{tabular}{|c|c|c|c|c|c|c|}
\hline Características & $\begin{array}{l}\text { Fatiga } \\
\text { n: } 49(\%)\end{array}$ & $\begin{array}{l}\text { No Fatiga } \\
\text { n: } 16(\%)\end{array}$ & $\begin{array}{l}\text { Depresión } \\
\text { n: } 25(\%)\end{array}$ & $\begin{array}{l}\text { No depresión } \\
\text { n: } 40(\%)\end{array}$ & $\begin{array}{l}\text { Disfunción } \\
\text { urinaria } \\
\text { n: } 27(\%)\end{array}$ & $\begin{array}{l}\text { No disfunción } \\
\text { urinaria } \\
\text { n: } 38(\%)\end{array}$ \\
\hline Mujer & $32(65,3)$ & $15(93,7)$ & $19(76)$ & $28(70)$ & $21(77,8)$ & $26(68,4)$ \\
\hline Trastornos del sueño & $18(36,73)$ & $3(8,75)$ & $16(64.0)$ & $5(12,5)$ & $10(37)$ & $11(28,9)$ \\
\hline Cefalea & $20(40,8)$ & $8(50)$ & $12(48)$ & $16(40)$ & $9(33)$ & $19(50)$ \\
\hline Disfunción sexual & $11(22,5)$ & $2(12,5)$ & $11(44)$ & $2(5)$ & $7(25)$ & $6(15)$ \\
\hline Disfunción anorectal & $15(30,61)$ & $3(18,7)$ & $10(40)$ & $8(20)$ & $10(37)$ & $8(21,1)$ \\
\hline $\begin{array}{l}\text { EDSS actual } \\
\text { mediana (RIQ) }\end{array}$ & $2(1-6)$ & $2(1-2,5)$ & $2(2-6)$ & $2(1-4)$ & $4(2-6)$ & $1(1-3)$ \\
\hline $\begin{array}{l}\text { Duración años de } \\
\text { enfermedad mediana }\end{array}$ & $\begin{array}{l}9 \text { (4-20) } \\
\text { (RIQ) }\end{array}$ & $6,5(3-1,5)$ & $9(6-20)$ & $6,5(2-15)$ & $11(6-21)$ & $4,5(2-12)$ \\
\hline
\end{tabular}

Nota: EDSS: escala de discapacidad EDSS; RIQ: rango Intercuartílico.

Fuente: autores. 
Un tercio de nuestros pacientes presenta algún síntoma relacionado con trastorno del sueño; el que más se presenta es el insomnio y un tercio de ellos presenta fatiga, lo que nos lleva a pensar que estos síntomas están interrelacionados y que una condición puede contribuir a la presentación de la otra. En cuanto a las patologías, la apnea obstructiva del sueño se encontró en un $4 \%$ y en un $2 \%$ para trastorno del sueño REM, cifras menores a las reportadas en otros estudios. A pesar de que en la literatura se menciona que la narcolepsia se puede presentar (39), ninguno de nuestros pacientes la padece.

El riesgo de presentar EM y depresión se ha estimado como alto, hasta un $50 \%$ en los pacientes la pueden presentar comparado con la población general, que es del 10 al 15 $\%$ (40); la prevalencia estimada de los síntomas depresivos es del $30 \%$ (41). En los pacientes del hospital, el $39 \%$ los presentan y se encontró que más de la mitad presenta concomitantemente algún trastorno del sueño.

Hallazgos en los estudios de cefalea y EM son diversos. Se ha encontrado una prevalencia global de cefalea del 53 $\%$, pero, al clasificarla, las cifras difieren. La migraña se ha reportado en un $31 \%$ y la cefalea tensional en un 14 $\%$ (17). En un segundo estudio se encontró un 16,3\% para migraña y un $24 \%$ con cefalea tensional (42). Asimismo, en algunos artículos se ha reportado que el uso de interferón puede inducir o empeorar la cefalea (43), aproximadamente de un 2 al $41 \%$ (44), lo que no se ha evidenciado con otros medicamentos modificadores de la enfermedad (18).

En nuestros pacientes la cefalea no se encontró relacionada con la presentación de otros síntomas, ni con el tiempo de evolución o el grado de discapacidad, y el tipo de cefalea que predomina es la cefalea tensional. 3 pacientes presentaron cefalea secundaria al manejo con interferón, esto es importante ya que cada tipo de cefalea se maneja de manera diferente y hay que verificar que no sea secundaria a la aplicación de los medicamentos modificadores de la enfermedad.

Otro síntoma que contribuye a la morbilidad de la enfermedad es la disfunción del tracto urinario; hay una gran variedad de síntomas presentados, dentro de los cuales tenemos síntomas obstructivos e irritativos. La prevalencia de la urgencia urinaria va de un 32 al $86 \%$, el aumento de la frecuencia del 31 al $85 \%$ y la incontinencia del 37 al 72 $\%$ (45); el vaciamiento incompleto y la obstrucción urinaria ocurren de un 34 al $79 \%$ (21).
En nuestra investigación encontramos una menor frecuencia de presentación; la mayoría de síntomas presentados son irritativos. Asimismo, es importante resaltar que solo un paciente presentó sintomatología obstructiva que difiere de lo reportado en la literatura.

La disfunción sexual reportada es del 50 al $90 \%$ para los hombres (46) y de un 40 al $80 \%$ en las mujeres (47); en otros artículos se encontró una frecuencia más alta en mujeres que en los hombres $(47,48)$. Estos resultados difieren totalmente de los nuestros, ya que solo un pequeño porcentaje de nuestros pacientes $(20 \%)$ presentan problemas con su sexualidad y se presenta más en el sexo femenino. De igual manera, se evidencian los mismos hallazgos para disfunción anorrectal, pues se encontró que tiene una baja frecuencia en nuestra población, comparada con otras series que van del 17 al $94 \%$ (49).

Por lo anteriormente descrito, se considera que cada uno de los síntomas mencionados puede estar asociado no solo a la EM como tal, sino también a sus características, como el de la progresión de la enfermedad y el grado de discapacidad. Esto podría afectar el desempeño en la vida cotidiana, sus relaciones interpersonales con la pareja y los familiares, llevando a un deterioro de la calidad de vida o pérdida de sus empleos. Por ello, hacer la descripción de todos estos hallazgos fue de suma importancia, ya que, así, se abarcará de una manera más amplia esta patología.

\section{CONCLUSIONES}

Se encontró que, al tener fatiga y depresión, a su vez, se presentan otros síntomas como los trastornos del sueño y disfunción sexual. Se observó que estos se presentan en más alta frecuencia con mayor tiempo de evolución de la enfermedad. Algunos de los resultados difieren totalmente de la literatura, como lo es la frecuencia de presentación de disfunción sexual y anorrectal. La disfunción del tracto urinario es el único que se relaciona con mayor grado de discapacidad.

Todo lo anterior conlleva a proponer una exhaustiva exploración, interrogatorio adecuado e intervención temprana de cada uno estos síntomas para mejorar la calidad de vida de nuestra población.

\section{Conflictos de intereses}

Los autores manifiestan no tener conflictos de intereses en este estudio. 


\section{REFERENCIAS}

1. Carretero Ares JL, Bowakim Dib W, Acebes Rey JM. Actualización: esclerosis múltiple. Medifam. 2001;11:30-43.

2. Ebers GC. Environmental factors and multiple sclerosis. The Lancet Neurology. 2008;7(3):268-77.

3. Huang WJ, Chen WW, Zhang X. Multiple sclerosis: Pathology, diagnosis and treatments. Exp Ther Med. 2017;13(6):3163-6.

4. Ontaneda D, Thompson AJ, Fox RJ, Cohen JA. Progressive multiple sclerosis: prospects for disease therapy, repair, and restoration of function. Lancet. 2017;389(10076):1357-66.

5. Sá MJ. Physiopathology of symptoms and signs in multiple sclerosis. Arq Neuropsiquiatr. 2012;70(9):733-40.

6. Covo P. Introducción a la historia de la esclerosis múltiple. Acta Neurológica Colombiana. 2015;31:119-24.

7. Browne P, Chandraratna D, Angood C, Tremlett H, Baker C, Taylor BV, et al. Atlas of Multiple Sclerosis 2013: A growing global problem with widespread inequity. Neurology. 2014;83(11):1022-4.

8. Toro J, Cárdenas S, Fernando Martínez C, Urrutia J, Díaz C. Multiple sclerosis in Colombia and other Latin American Countries. Multiple Sclerosis and Related Disorders. 2013;2(2):80-9.

9. Jiménez-Pérez CE, Zarco-Montero LA, Castañeda-Cardona C, Otálora Esteban M, Martínez A, Rosselli D. Estado actual de la esclerosis múltiple en Colombia. Acta Neurol Colomb. 2015;31(4):385-90.

10. Sánchez JL, Aguirre C, Arcos-Burgos OM, Jiménez I, Jiménez M, León F, et al. [Prevalence of multiple sclerosis in Colombia]. Rev Neurol. 2000;31(12):1101-3.

11. Polman $\mathrm{CH}$, Reingold SC, Banwell B, Clanet M, Cohen JA, Filippi M, et al. Diagnostic criteria for multiple sclerosis: 2010 revisions to the McDonald criteria. Ann Neurol. 2011;69(2):292-302.

12. Brownlee WJ, Hardy TA, Fazekas F, Miller DH. Diagnosis of multiple sclerosis: progress and challenges. Lancet. 2017;389(10076):1336-46.

13. Kurtzke JF. Rating neurologic impairment in multiple sclerosis: an expanded disability status scale (EDSS). Neurology. 1983;33(11):1444-52.

14. Motl RW, Learmonth YC. Neurological disability and its association with walking impairment in multiple sclerosis: brief review. Neurodegener Dis Manag. 2014;4(6):491-500.

15. Rae-Grant AD. Unusual symptoms and syndromes in multiple sclerosis. Continuum (Minneap Minn). 2013;19(4 Multiple Sclerosis):992-1006.

16. Khan F, Amatya B, Galea M. Management of fatigue in persons with multiple sclerosis. Front Neurol. 2014;5:177.

17. Terlizzi R, Merli E, Buccellato E, Giannini G, Favoni V, Pierangeli G, et al. P037. Headache in multiple sclerosis: prevalence and clinical features in a case control-study. J Headache Pain. 2015;16(Suppl 1):A83.

18. Putzki N, Pfriem A, Limmroth V, Yaldizli O, Tettenborn B, Diener HC, et al. Prevalence of migraine, tension-type headache and trigeminal neuralgia in multiple sclerosis. Eur J Neurol. 2009;16(2):262-7.

19. Marrie RA, Reingold S, Cohen J, Stuve O, Trojano M, Sorensen PS, et al. The incidence and prevalence of psychiatric disorders in multiple sclerosis: A systematic review. Mult Scler. 2015.
20. Strober LB. Fatigue in multiple sclerosis: a look at the role of poor sleep. Front Neurol. 2015;6:21.

21. Dillon BE, Lemack GE. Urodynamics in the evaluation of the patient with multiple sclerosis: when are they helpful and how do we use them? Urol Clin North Am. 2014;41(3):439-44, ix.

22. Munteis E, Andreu M, Téllez MJ, Mon D, Ois A, Roquer J. Anorectal dysfunction in multiple sclerosis. Mult Scler. 2006;12(2):215-8.

23. Krupp LB, Alvarez LA, LaRocca NG, Scheinberg LC. Fatigue in multiple sclerosis. Arch Neurol. 1988;45(4):435-7.

24. Bulbena A BG, Fernández de Larrinoa P. Medición Clínica en Psiquiatría y Psicología. Madrid: Masson SA; 2000.

25. Hamilton M. A rating scale for depression. J Neurol Neurosurg Psychiatry. 1960;23:56-62.

26. Ramos-Brieva J, Cordero Villafafila A. Validación de la versión castellana de la escala de Hamilton para la depresión. Actas Luso Esp Neurol Psiquiatr Cienc Afines. 1986;14:324-34.

27. Sanders AS, Foley, F. W., LaRocca, N. G., Zemon, V. The Multiple Sclerosis Intimacy and Sexuality Questionnaire-19 (MSISQ-19). Sexyality and Disability. 2000;18(Issue 1):3-26. Available from: http://eds.b.ebscohost.com.ezproxy.unal.edu. $\mathrm{co} / \mathrm{eds} /$ detail $/$ detail ?vid $=2 \&$ sid $=$ cecfa041-4655-44f8-a8b1f9a6aca3d764@sessionmgr110\&hid=121\&bdata=Jmxhbmc9 ZXMmc210ZT11ZHMtbGl2ZQ==\#AN=11303668\&db=a9h.

28. Headache Classification Committee of the International Headache Society (IHS). The International Classification of Headache Disorders, 3rd edition (beta version). Cephalalgia. 2013;33(9):629-808.

29. Cores EV, Vanotti S, Burin DI, Politis DG, Villa A. [Factors associated to the work situation of patients with multiple sclerosis]. Rev Neurol. 2014;58(4):175-83.

30. Pearson JF, Alla S, Clarke G, Mason DF, Anderson T, Richardson A, et al. Multiple Sclerosis impact on employment and income in New Zealand. Acta Neurol Scand. 2016.

31. Cadden M, Arnett P. Factors Associated with Employment Status in Individuals with Multiple Sclerosis. Int J MS Care. 2015;17(6):284-91.

32. Marrie RA, Cutter GR, Tyry T, Cofield SS, Fox R, Salter A. Upper limb impairment is associated with use of assistive devices and unemployment in multiple sclerosis. Mult Scler Relat Disord. 2017;13:87-92.

33. Pfleger CC, Flachs EM, Koch-Henriksen N. Social consequences of multiple sclerosis. Part 2. Divorce and separation: a historical prospective cohort study. Mult Scler. 2010;16(7):87882.

34. Marrie RA, Reider N, Cohen J, Trojano M, Sorensen PS, Cutter $G$, et al. A systematic review of the incidence and prevalence of sleep disorders and seizure disorders in multiple sclerosis. Mult Scler. 2014.

35. Gasparini S, Ferlazzo E, Ascoli M, Sueri C, Cianci V, Russo C, et al. Risk factors for unprovoked epileptic seizures in multiple sclerosis: a systematic review and meta-analysis. Neurol Sci. 2017;38(3):399-406.

36. Fiest KM, Fisk JD, Patten SB, Tremlett H, Wolfson C, Warren $\mathrm{S}$, et al. Fatigue and Comorbidities in Multiple Sclerosis. Int J MS Care. 2016;18(2):96-104. 
37. Pokryszko-Dragan A, Bilińska M, Gruszka E, Biel Ł, Kamińska $\mathrm{K}$, Konieczna K. Sleep disturbances in patients with multiple sclerosis. Neurol Sci. 2013;34(8):1291-6.

38. Nagaraj K, Taly AB, Gupta A, Prasad C, Christopher R. Depression and sleep disturbances in patients with multiple sclerosis and correlation with associated fatigue. J Neurosci Rural Pract. 2013;4(4):387-91.

39. Lunde HM, Bjorvatn B, Myhr KM, Bø L. Clinical assessment and management of sleep disorders in multiple sclerosis: a literature review. Acta Neurol Scand Suppl. 2013(196):24-30.

40. Alsaadi T, El Hammasi K, Shahrour TM, Shakra M, Turkawi L, Mudhafar A, et al. Prevalence of Depression and Anxiety among Patients with Multiple Sclerosis Attending the MS Clinic at Sheikh Khalifa Medical City, UAE: Cross-Sectional Study. Mult Scler Int. 2015;2015:487159.

41. Boeschoten RE, Braamse AM, Beekman AT, Cuijpers P, van Oppen P, Dekker J, et al. Prevalence of depression and anxiety in Multiple Sclerosis: A systematic review and meta-analysis. J Neurol Sci. 2017;372:331-41.

42. Möhrke J, Kropp P, Zettl UK. Headaches in multiple sclerosis patients might imply an inflammatorial process. PLoS One. 2013;8(8):e69570.
43. Patti F, Nicoletti A, Pappalardo A, Castiglione A, Lo Fermo $\mathrm{S}$, Messina $\mathrm{S}$, et al. Frequency and severity of headache is worsened by Interferon- $\beta$ therapy in patients with multiple sclerosis. Acta Neurol Scand. 2012;125(2):91-5.

44. La Mantia L. Headache and multiple sclerosis: clinical and therapeutic correlations. Neurol Sci. 2009;30 Suppl 1:S23-6.

45. Sadiq A, Brucker BM. Management of neurogenic lower urinary tract dysfunction in multiple sclerosis patients. Curr Urol Rep. 2015;16(7):44.

46. Prévinaire JG, Lecourt G, Soler JM, Denys P. Sexual disorders in men with multiple sclerosis: evaluation and management. Ann Phys Rehabil Med. 2014;57(5):329-36.

47. Zamani M, Tavoli A, Yazd Khasti B, Sedighimornani N, Zafar M. Sexual Therapy for Women with Multiple Sclerosis and Its Impact on Quality of Life. Iran J Psychiatry. 2017;12(1):58-65.

48. Celik DB, Poyraz E, Bingöl A, Idiman E, Ozakbaş S, Kaya D. Sexual dysfunction in multiple sclerosis: gender differences. J Neurol Sci. 2013;324(1-2):17-20.

49. Nusrat S, Gulick E, Levinthal D, Bielefeldt K. Anorectal dysfunction in multiple sclerosis: a systematic review. ISRN Neurol. 2012;2012:376023.W 